\title{
3D Catalogue based on Augmented Reality in Android Operating System
}

\author{
Natalia Chandra, Fredy Purnomo, Michael Yoseph Ricky, Christianto Leonard, Arvin Verdian, Hendrick \\ School of Computer Science, Bina Nusantara University, Jakarta, 11480, Indonesia
}

\begin{abstract}
Along with the continued development of Smartphone and the era of globalization, human needs are increasing for technology. They continue to look for ways that can ease their lives and it is up to the explanation of the visualization that closer to the actual visualization and support fast, precise and clear retrieval of interactive information. In order to realize the visualization, augmented reality technology is being used. Augmented reality is a technology that uses camera technology to recognize real world, images, objects, and environments and superimposes virtual information and data onto reality in real time. It combines a virtual object into two or three dimensions in a real three-dimensional environment. By developing an application with augmented reality, it can help user to look more detail into a product and give advantages to company to save the brochure production cost. The development of this application is using client-side and server-side, where the client-side will be run on android smartphone and server-side using a php based web service. The goal of this paper is to develop a 3D catalogue that helps to depict a product in interactive ways with zoom, rotate, choose color, and animation features.
\end{abstract}

Keywords: Augmented Reality, Android, Catalogue, Marker, Smartphone, 3D Model.

\section{Introduction}

Many companies and organization nowadays promote their products conventionally such as advertising in the media, posters or brochures, catalog and those cost a lot of money. Augmented reality is a type of computer vision that uses camera technology to recognize real world images, objects, and environments and superimposes virtual information and data onto reality in real time. It turns the smart devices into a lens for adding video, audio, 2-D content, 3-D content and location-based information [1]. Carmigniani describe about the technology that used to do augmented reality, which are computer vision that divided into 2, featured based and model based, AR devices like display, input devices, tracking, and computer, AR interfaces like tangible AR interfaces, collaborative AR interfaces, hybrid AR interfaces, and multimodal AR interfaces, and AR Systems [2]. Schmalstleg, Langlotz, dan Billinghurst describe about augmented reality 2.0 concept, which are the combination of augmented reality 2.0 and web 2.0, where the augmented reality itself is developed in mobile [6].

In the last few years, many developers tried to apply augmented reality technology to give interactive interaction for user in terms of information search, especially in the smartphone technology. Based on International Data Corporation (IDC) shown in fig. 1, android continue to dominate the global smartphone market, with over 255 million units shipped and $85 \%$ of the market share in the second quarter of 2014 [3].
By looking the development of both technologies, many companies use those to reduce production cost and increase the product sale. Using augmented reality, allow customer to look the product picture more real so it can represent the product intact. Combining augmented reality with the internet can connect customer and companies anytime and anywhere with Smartphone.

The next section describes about reporting tool requirements. Section III describes the comparison of reporting tools. The detail of architecture is described in section IV. The last section is evaluation and summary.

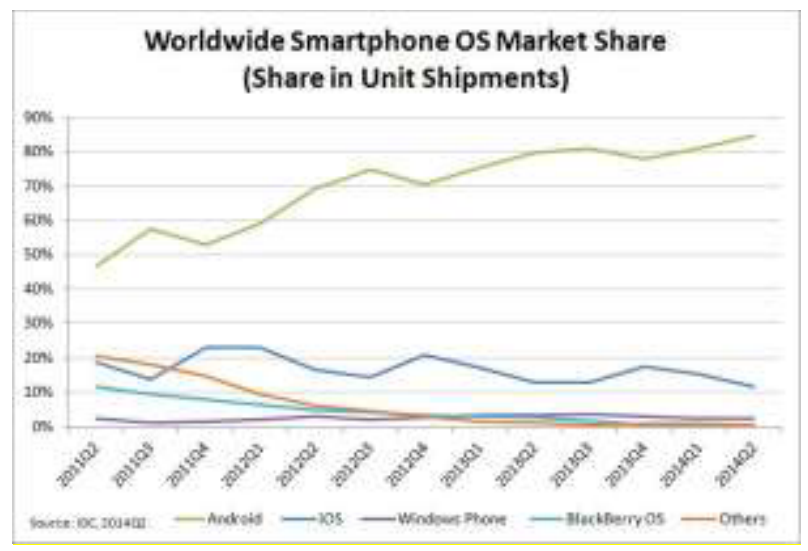

Fig.1: WorldWide Smartphone Market Share 


\section{Comparison between similar augmented reality application}

In this section, we will compare some recent augmented reality application that developed before, which are: Brother AR and Go Sweat Go Ion.

\subsection{Brother AR}

This is an augmented reality application that published by Brother International Singapore Pte Ltd as an interactive way to promote their product.

The application was claimed as the first 3D mobile augmented reality (AR) application to experience printers and Multi-Function Centre (MFC) at a different level from Brother. This is a free application that can be downloaded from play store. In the application, there is a virtual setup feature that allow user to simulate setting up the printer at home or office and give the idea how the product will fit into a space [4]. Fig. 2 shows the user interface of Brother AR application.

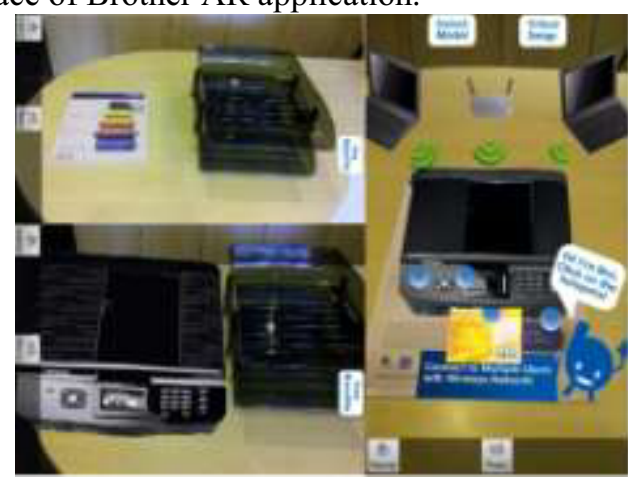

Fig.2: Brother AR Application

\subsection{Go Sweat Go Ion.}

This is an application that published by Amerta Indah Otsuka Company as a sole proprietor for pocari sweat drinks in Indonesia. This application is made for the purpose of giving education to society of how important exercise and consume their drink.

Besides that, there are a lot contents inside that require the user to keep moving, such as Capturion game that allow user to catch the ions using hand [5]. Fig.3 shows the user interface of Go Sweat Go Ion application.

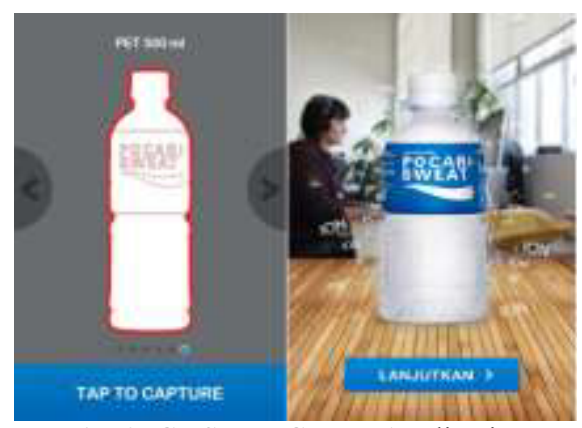

Fig. 3: Go Sweat Go Ion Application

\section{Augmented reality 3D catalogue detail and architecture}

The application will be developed on client-side and server-side where the client-side will be run on Smartphone and the server-side will be run on a web service that connect the admin and database server. All the features are developed to synchronize the data between the client-side to the database so that the application can be run offline and the feature will run online to download the data from database server.

In this section, there are five features that will be explained which are views the 3D model, choose the colour, zoom, rotate, and run the 3D model.

\subsection{View 3D Model}

In this feature, user can view the $3 \mathrm{D}$ model by scan the marker through their smartphone camera. Fig. 4 shows the detail architecture on how this feature works:

a. User opens the application and point the smartphone camera to the 2D marker.

b. The $2 \mathrm{D}$ marker will be search by the sensor ID in database.

c. If the $2 \mathrm{D}$ marker detected by the server, the system will get the $3 \mathrm{D}$ object and texture. The user can view the $3 \mathrm{D}$ model through their smartphone.

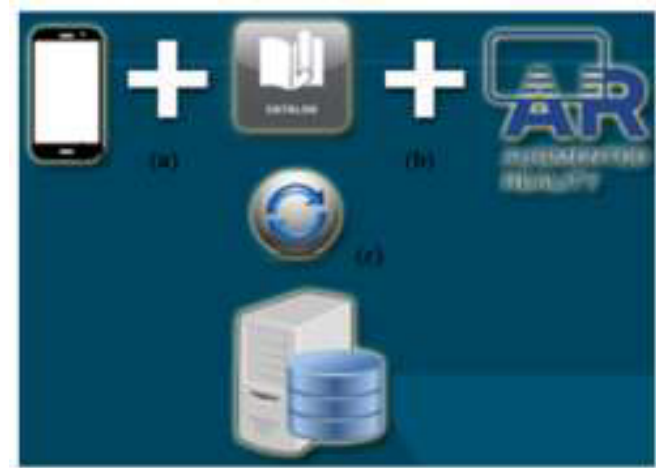

Fig. 4: View 3D Model Architecture

Fig. 5 shows the 3D model in smartphone view after user scan the 2D marker. There is guidance for user on how to use the features.

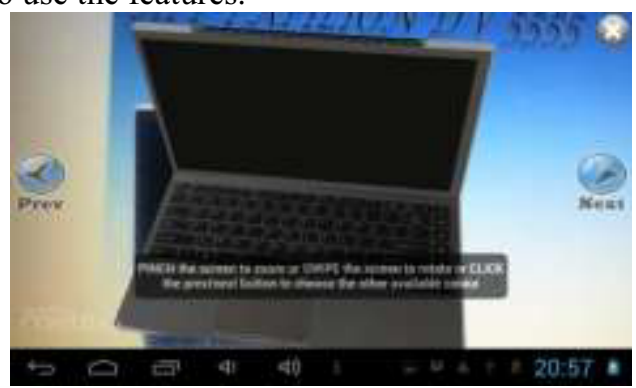

Fig. 5: View 3D Model in Smartphone View

\subsection{Choose The Colour of 3D Model}

In this feature, users are able to look available different colours of a product. The architecture of this feature is start when user able to look the 3D model on the screen like shown in fig.5, the system will get the 3D object and all the textures based on the product. The detail architecture shows in table 1 , the alt-step explain about 
when the product only have one colour, then system only show the available colour to user.

Table 1: Change Colour Detail Architecture

\begin{tabular}{|l|l|}
\hline Actor Action & \multicolumn{1}{|c|}{ System Response } \\
\hline Step-1 & $\begin{array}{l}\text { Step-2 } \begin{array}{l}\text { Get the 3D texture } \\
\text { based on the } \\
\text { product ID. } \\
\text { and Next button. }\end{array} \\
\text { Put the texture to } \\
\text { 3D model and show } \\
\text { the 3D model to } \\
\text { user. }\end{array}$ \\
\hline Alt step-2 & $\begin{array}{l}\text { Step-3 there is no texture on that } \\
\text { product, then the 3D model will } \\
\text { not change colour. }\end{array}$ \\
\hline
\end{tabular}

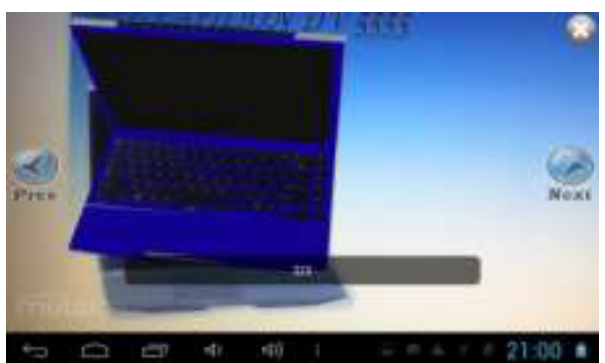

Fig. 6: Choose Colour 3D Model in Smartphone View

Fig. 6 shows the user interface in smartphone view. There are two buttons which are prev and next, the users are able to choose the colour of a product by click the prev and next button in the smartphone screen.

\subsection{Zoom the 3D Model}

In this feature, users are able to look the product in different sizes by pinch action in smartphone screen. The detail architecture shows in table 2 .

Table 2: Zoom 3D Model Detail Architecture

\begin{tabular}{|c|c|c|c|}
\hline & ctor Action & \multicolumn{2}{|c|}{ System Response } \\
\hline Step-1 & $\begin{array}{l}\text { Users do the } \\
\text { pinch in or pinch } \\
\text { out to the screen. }\end{array}$ & Step-2 & $\begin{array}{l}\text { Do the zoom in or } \\
\text { zoom out and show } \\
\text { it to the } 3 \mathrm{D} \text { model. }\end{array}$ \\
\hline
\end{tabular}

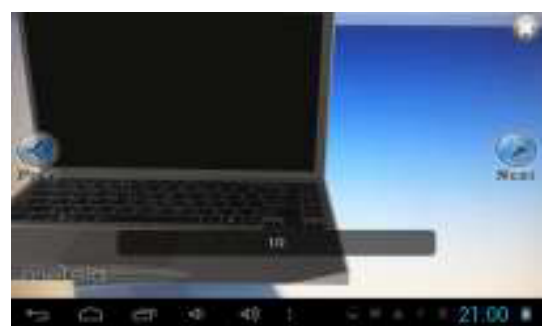

Fig. 7: Zoom the 3D Model in Smartphone View (Pinch Out)

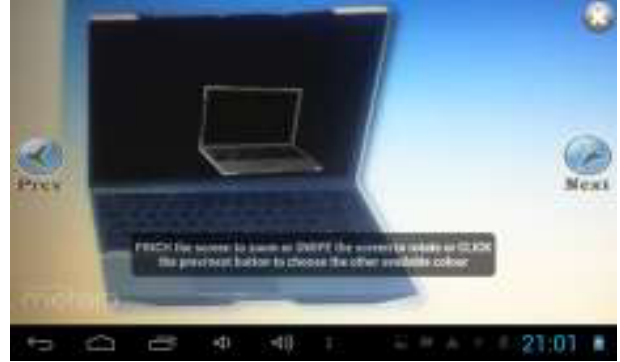

Fig. 8: Zoom the 3D Model in Smartphone View (Pinch in)

Fig. 7 shows the user interface how the 3D object is zooming out by pinch out and Fig. 8 shows the user interface how the $3 \mathrm{D}$ object is zooming in by pinch in, in the smartphone view.

\subsection{Rotate the 3D Model}

In this feature, users are able to rotate the 3D model with swipe action to smartphone screen. The rotation process will focus on the $\mathrm{X}$ and $\mathrm{Y}$ axis. The process will be:

a. If user swipe up the 3D model, then the system will add the $\mathrm{X}$-axis $(\mathrm{x}++)$ like shows in fig. 9.

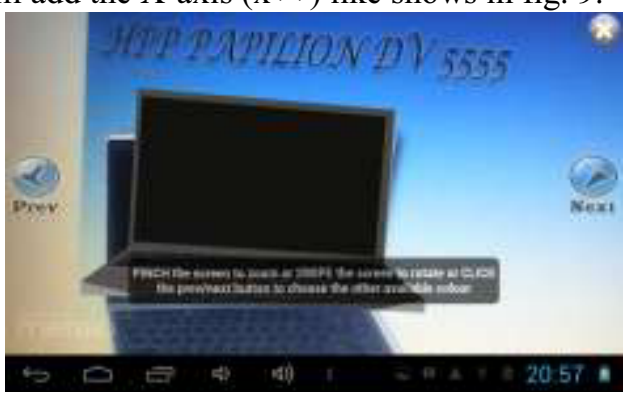

Fig. 9: Rotate 3D Model Swipe Up

b. If user swipe down the $3 \mathrm{D}$ model, the system will minus the $\mathrm{X}$-axis (x--) like shows in fig. 10.

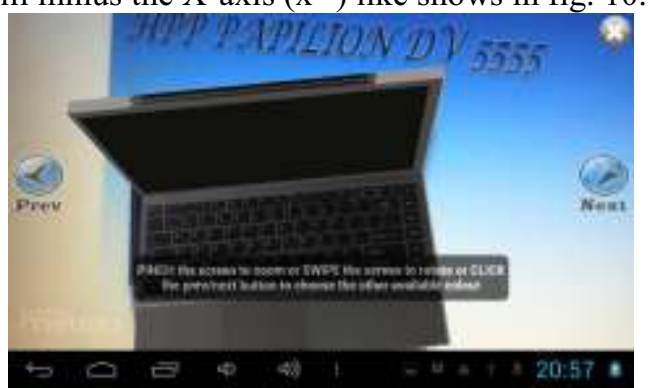

Fig. 10: Rotate 3D Model Swipe Down

c. If user swipe left the 3D model, the system will add the $\mathrm{Y}$-axis $(\mathrm{y}++)$ like shows in fig. 11.

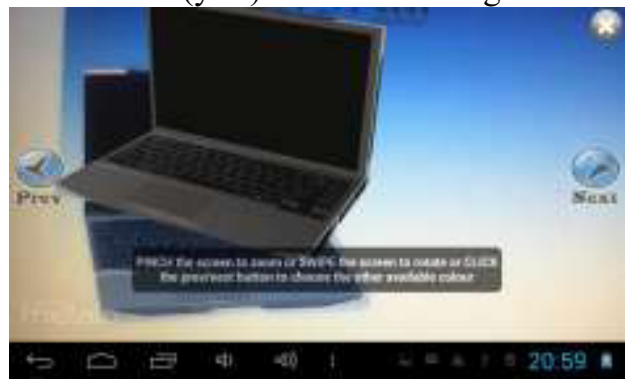

Fig. 11: Rotate 3D Model Swipe Left 
d. If user swipe right the 3D model, the system will minus the $\mathrm{Y}$-axis (y--) like shows in fig. 12.

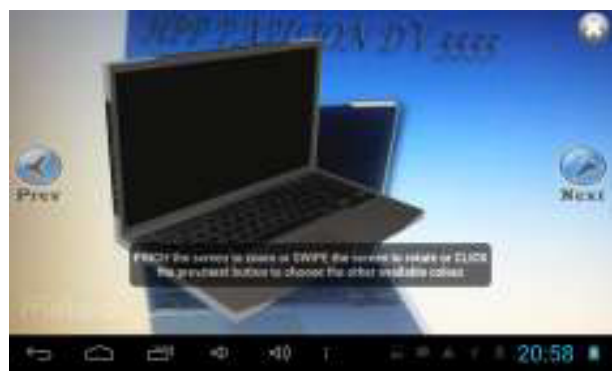

Fig. 12: Rotate 3D Model Swipe Right

\subsection{Run the 3D Model Animation}

In this feature, users are able to view the animation of a 3D model by click on to it. The system will search through the product ID, if there is available animation on the product, the system will return it to the user. The detail architecture shows in Table 3, how the user action and the system response. The alt-step is explained about when there is no animation data, the system will show nothing to the smartphone screen.

Table 3: Run 3D Model Animation Detail Architecture

\begin{tabular}{|c|c|c|c|}
\hline \multicolumn{2}{|c|}{ Actor Action } & \multicolumn{2}{|c|}{ System Response } \\
\hline Step-1 & $\begin{array}{l}\text { User click or } \\
\text { touch on 3D } \\
\text { model. }\end{array}$ & Step-2 & $\begin{array}{l}\text { Run the animation } \\
\text { that save on } 3 \mathrm{D} \\
\text { model. }\end{array}$ \\
\hline \multicolumn{4}{|c|}{$\begin{array}{l}\text { Alt step-2 If there is no animation saved in 3D model then } \\
\text { there is no animation will be run. }\end{array}$} \\
\hline
\end{tabular}

\section{Conclusions}

Based on research of two applications, Brother AR and Go Sweat Go Ion features. Both of applications have similar features which are view 3D model, animation and markerless. 3D Catalogue application give more features and make user able to look the product in different perspective by using zoom, rotate, and color chooser features. Brother AR and Go Sweat Go Ion application focused on one product, but using 3D catalogue can give the company to promote more of their products. There are several future work that will be done in the future by authors to make 3D catalogue application more complete such as develop it in another platform, connect to company social media and notification to user every time company has new product.

\section{References}

1. Augmented Reality, http://www.metaio.com/technology/, Metaio GmbH 2014.

2. J. armigniani, B. Furht, M. Anisetti, P. Ceravolo, E. Damiani, and M. Ivkovic, Augmented Reality
Technology, Systems and Application. Springer Science+Business Media, 51, 341-377, (2010).

3. IDC: International Data Corporation, Smartphone OS Market Share, Q2 2014, http://www.idc.com/

4. Brother AR: http://play.google.com, Brother International Singapore, 2012.

5. Go Sweat Go Icon: http://play.google.com, PT. Amerta Indah Otsuka, 2013.

6. D. Schmalstleg, T. Langlotz, and M. Billinghurst, Augmented Reality 2.0. Springer-Verlag/Wien, 10, 13-37, (2011). 